\title{
Spinal TB: Impact of Research Evidence on Clinical Practice
}

\author{
Anil K Jain \\ Department of Orthopaedics, \\ University College of Medical Sciences and GTB Hospital, New Delhi.
}

\begin{abstract}
The evidence generated while treating the patients is the key for growth of science. Finding answers to series of research questions spread over many years may change the clinical practice. This presentation is based on 25 research questions, 44 publications while treating 3300 patients over last 28 years ( 1990-2017) which has substantially changed the objective of treatment in spinal tuberculosis (TB) from healing of lesion with sequelae of spinal deformity and paraplegia to achieving healed status with near normal spine.
\end{abstract}

Three cases of late-onset paraplegia were evaluated (1990) by newly introduced MRI. The syringohydromyelia and severe cord atrophy were attributed as the cause of paraplegia. We conducted a series of prospective studies to define and correlate MRI observations on spinal cord in paraplegia and followed the treatment outcomes. The cord edema, myelomalacia, cord atrophy and syringomyelia were observed in cases with neural complications. The patients with cord edema and liquid compression are predictor for neural recovery, while dry lesions and myelomalacia for poor neural recovery. The mild cord atrophy was consistent with neural recovery while severe cord atrophy with sequalae of neural deficit. Upto $76 \%$ canal encroachment was found compatible with intact neural state. Spinal deformity in TB spine is better prevented than treated. The contagious vertebral body disease with intact disc spaces, subperiosteal and paravertebral, septate abscesses, intra-osseous and intraspinal abscesses are considered features of spinal TB and resolution of abscess and fatty replacement is characteristic of healing. The clinicoradiological predictors for diagnosing spinal TB in predestructive disease were defined. Only 35\% patients achieved healed status on MRI by DOTS regimen at 8 months, Hence, it is unscientific to stop antitubercular treatment (ATT) at fixed time schedule. The criteria to suspect multi-drug resistant (MDR)-TB and guide to treatment were defined.

Residual Kyphotic deformity in spine TB produces severe proximal/distal degeneration of spine and/or late-onset paraplegia. We correlated the final kyphosis with initial vertebral body (VB) loss, where 1.5 VB height loss will produce 600 spinal deformity or more, hence surgical correction of spinal deformity is indicated. The surgical steps of kyphotic deformity correction are: anterior corpectomy, posterior column shortening, instrumented stabilization, anterior gap grafting and posterior fusion in a single stage and sequentially. The surgical incision of costo-transversectomy was modified so that kyphosis correction and posterior Hartshill instrumentation can be performed simultaneously. The retroperitoneal extrapleural approach for dorsolumbar spine was described. Meta-analysis of spinal instrumentation in TB spine established the lack of defined indication of instrumented stabilisation. Panvertebral/ long segment disease, kyphotic deformity correction are listed as indications of

Correspondence: Dr. Anil K Jain, Director-Professor and Head, Department of Orthopaedics, University College of Medical Sciences and GTB Hospital, New Delhi-110095, India. Mob: 9811604663. Email:profakjain@gmail.com.

COL. SANGHAM LAL MEMORIAL ORATION delivered during NAMSCON 2017 at Sri Guru Ram Das Institute of Medical Sciences \& Research, Sri Amritsar, Punjab. 
instrumented stabilisation in TB spine. The end point of treatment in spinal TB still eludes us to resolve the optimum duration of ATT regimen. The PET scan may be used to define it. We believe if a clinician works slow and steady on a series of research questions and by sustained focused efforts can change the clinical practice. We after this sustained research work could contribute in framing Bone and Joint TB guidelines and publish as monograph.

Keywords: Bone tuberculosis, TB spine, antitubercular therapy, kyphotic deformity.

\section{Introduction}

The medicine has evolved as an art based on experience of observing and treating the persons in pain. In ancient time it was hypothesized that disease occurs due to curse of supernatural forces. Gradually over the years since (3000 years) ago a scientific basis of disease was found to execute treatment predictably. The persons involved in executing treatment remedies developed treatment practices over centuries by continued observations. They transmitted this experience by passing them to their disciples to cultivate expertise. Currently the treatment practices are not based on expert opinion. It is based on evidence generated by researches conducted over a series of similar patients in different parts of the world and published for scientific scrutiny and its continued validation.

The human body is complicated, where the biological processes (physiological and pathological) are affected by multiple factors. The response of each human body to inciting agents to cause disease and the response to treatment is variable. That is why suspecting a diagnosis from the symptomatology, making a diagnosis with the help of relevant investigations, defining affliction and extent of damage to organ(s) or body parts and determining drugs, its volume and duration of treatment to affect a cure is an art. The intervention and anticipating the treatment response is described as "artistic application of science". The diagnosis and treatment executed based on more and more evidence generated from research is important to predictably cure the ailment.
The modern (allopathy) medicine was started in Europe, Ayurveda in the Indian subcontinent and Chinese medicine at about the same time (3000 years). All three were based on religious and philosophical assumptions and thus the treatment executed was empiric. The practical medical experiences by the clinicians were passed down orally through numerous generations. "Sushruta Samhita" and Charak Samhita were written around 6th century BC. Sushruta Samhita detailed surgical techniques of making incisions, probing, foreign body extractions, alkali and thermal cauterizations, tooth extractions, caesarean section, and other surgical procedures (1). Thus, Ayurveda was far ahead of Western medicine in surgery.

Modern medicine also had a long history of pre-scientific stage of development. The clinicians practicing modern medicine applied proper scientific principles over the years and discarded old superstitious ideas with no genuine medical foundation. The observations were recorded and published in scientific journals, thus modern medicine evolved and developed to what it is today. Ayurveda did not evolve scientifically, hence continued in prescientific stage. Currently word medicine is synonymous with modern medicine and Ayurveda and Chinese medicine are called alternative medicine.

Whenever a clinical problem is confronted by a clinician, he/she tries to identify the cause and evolve treatment strategy to treat it. The outcome of treatment when scrutinized and assessed on scientific parameters, becomes an evidence. The evidence generated on a series of similar patients (inclusion criteria's), evaluated 
on scientific parameters becomes the definite evidence and a research. When the generated evidence is published in peer-reviewed journals, it can be retrieved beyond human life and is available worldwide for posterity. It also becomes the foundation stone for future research and guiding principle for current treatment remedies.

\section{Spinal Tuberculosis}

Tuberculosis (TB) is as old as human being on mother earth and bacteria and human being have lived in symbiosis (2). The mycobacterium has been demonstrated by molecular methods in Egyptian mummies and are found in remains of iron age. The treatment of TB has evolved as the medical science evolved. The discovery of organism and antibiotic against mycobacterium has improved the outcome of treatment. Patients with TB spine in pre-antibiotic era were treated in sanatorium (by high protein diet, rest, fresh air, and sunshine) with the hope for natural quiescence of disease. Only few patients used to survive and remained crippled and rest used to die. The introduction of antitubercular drugs allowed the TB lesions to heal, thus the objective of treatment now changed to achieveing healing with residual sequlae of spinal deformity and neural complications. The development of diagnostics, imaging, surgical interventions, and instrumentations, anesthetic care, intensive care has improved and the objective of treatment to "healing of the lesion with near normal spine" (2).

\section{Treatment Practices-TB Spine (1988)}

The patients of TB spine without neural complications were treated by ambulant chemotherapy and by middle path regimen (surgery when indicated) with neural complications, on the contrary western centers were following universal surgical extirpation. Since the diagnosis was based on plain X-rays, hence the patients were reporting for the first time with kyphotic deformity. The healing of TB spine with residual kyphosis and/or sequalae of neural deficit was the expected outcome. There was a controversy on the optimum duration of antitubercular treatment (ATT) as well as the need/ rational of surgical intervention and instrumented stabilization. The kyphotic deformity was an unsolved issue in planning the management.

\section{Beginning of the Journey of TB Spine Research}

We encountered a case (1988) of late onset paraplegia (grade III) 20 years after initially being treated by anterolateral decompression (TB spine D3-4 with paraplegia). He had no symptoms of active disease of TB spine at this presentation. While analyzing the cause of late onset paraplegia, magnetic resonance imaging (MRI) scan, a new imaging modality at that time, available in an army establishment was performed. The spinal cord showed severe cord atrophy, and syringomyelia with healed vertebral lesion, severe kyphotic deformity and cord impingement by internal salient. Two other similar patients of late onset paraplegia also showed severe cord atrophy. Hence, we reported that severe cord atrophy and syringomyelia are attributed as the causes of late onset of paraplegia (3). These 3 cases stimulated us to explore MRI observations in TB spine with or without neural deficit: a) to diagnose spinal TB by MRI well before a deformity develops; b) diagnostic features of TB spine and features of healed disease by MRI; and c) defining MRI correlates of recovering/ non-recovering tuberculous paraplegia.

\section{MRI Diagnosis of TB Spine}

Forty-nine consecutive patients diagnosed clinico-radiologically and/or histology/fine needle aspiration cytology/AFB smear/molecular method (PCR) were enrolled. The MRI findings in these patients were analysed for diagnostic criteria for TB spine and 
healing changes (4). The tubercular lesions were more extensive on MRI than that appreciated on X-rays as mean vertebral body (VB) affection on X-rays was $2.61(\mathrm{n}=128 \mathrm{VB})$ and on MRI $3.2(n=161 \mathrm{VB})$. On MRI contiguous VB involvement, relative preservation of disc space, subligamentous paravertebral collection (low signal in T1WI and bright on T2WI), septate paravertebral collection, marrow edema, and epidural involvement (canal encroachment), intraosseous abscess, were considered diagnostic features of spinal TB. The patient not showing classical findings on MRI, but are suspected tubercular lesion (case of diagnostic dilemma) must be subjected for tissue diagnosis before starting ATT. The resolution of marrow edema and collection, fatty replacement of bone marrow, and resolution of cord signal intensity and absence of contrast enhancement were observed with healing/healed disease.

\section{Early Diagnosis of Spinal TB before Development of Deformity}

Spinal TB is a slowly developing disease. The bony lesion can only be appreciated if bone losses $40 \%$ of its calcium content (5). Thus radiological findings appear almost 3-4 months after the onset of disease. The patient continue to remain symptomatic during these 3-4 months. Since MRI could depict inflammation earlier, hence can be useful to diagnose TB spine in predestructive stage of disease. We defined the clinical or clinico-radiological predictors to suspect spinal TB lesion in early stage of disease. The patient with persistent localized back pain ( 3 months or more) with or without tenderness or spasm, with or without constitutional symptoms, with or without early radiological finding should be labeled as "observational spine" in the endemic zone for TB and need to be serially evaluated by X-rays. With subtle X-rays findings, the patient should be subjected to MRI. These X-ray findings of early suspicion will be variable depending on segment of spine affected $(6,7)$.

\section{Cervical spine}

The prevertebral soft tissue space (PVSTS) in cervical spine contains loose areolar tissue where inflammatory exudates collects when the underlying VB is affected by TB and can be appreciated as increase in PVSTS on X-rays The normal PVSTS space is described in millimeters, but it may be variable depending on height and built of the patient and particularly difficult to measure in digital X-rays. The normal PVSTS on lateral radiograph of the cervical spine $(n=300)$ was calculated and described in relation to the width of the underlying VB. Before the bifurcation of nasopharynx into esophagus and trachea, PVSTS is one third and after the bifurcation it is two third of the width of VB behind. In front of C7, it is always less than C6. In front of the upper dorsal spine it should be 8-10 $\mathrm{mm}$ away from the vertebra and should follow the contour of upper dorsal spine, i.e., concave interiorly (8). Any localized or generalized increase in PVSTS is an indirect indicator of pathology in underlying bones. On observing increase in PVSTS the underlying bone disease may be diagnosed on MRI. Below D4-D5 the intervertebral disc height can be very well-appreciated on plain X-rays. The disc height on plain X-ray at any level should be equal or more than the disc above except D12-L1 and L5-S1. The early reduction of disc height in a symptomatic patient can be considered a suspected case of TB spine and with MRI can be diagnosed in inflammatory stage of disease well before a spinal deformity develops. If the treatment is started in predestructive stage (inflammatory stage), it would heal with almost near normal spine.

\section{Tuberculous Myelopathy (Correlation of Clinical Course and MRI Observations)}

Sixty patients of TB spine with paraquadriplegia were serially evaluated by MRI, while on treatment and 143 MRI scans were analysed (9). The cord compression was of two types: a) Liquid compression, having low signal 
intensity on T1WI and bright signal on T2WI; b) Dry compression diagnosed as mixed extradural collection with the heterogeneous signal in T1WI and T2WI. The changes observed on spinal cord were: (i) cord edema observed as diffuse hyperintensity in T2WI and hypointensity on T1WI; (ii) myelomalacia as patchy hyperintensity in T2WI and hypointensity in T1WI; (iii) cord atrophy observed as a reduction in cord volume; and (iv) syringomyelia as dilatation of the central canal.

The predominant liquid compression, preserved spinal cord volume, and cord edema was observed in all those patients who recovered neurologically after treatment ( non-operative or surgical) provided the patient does not show any imaging features of instability (like panvertebral disease).

The patients showing mixed extradural compression (dry lesion) and thick duraarachnoid complex with diseased tissue encircling the spinal cord with features of myelomalacia are unlikely to show neural recovery and are indication for early surgical decompression.

\section{Canal Encroachment}

Intraspinal encroachment by granulation tissue is also observed in MRI scan even when patient has no neural deficit. MRI/CT scan of 16 cases of TB spine with no neural complications were enrolled (10). The area of the spinal cord and the spinal canal were calculated at the level of maximum compression in axial view. Upto $76 \%$ canal encroachment was observed in these patients and they did not have imaging features of instability ( $p$ athologic a 1 subluxation/dislocation). The cord compression in the TB spine is gradual and spinal cord has time to adapt to gradually building cord compression. The neural deficit may develop at lesser canal compromised in the presence of spinal instability.

\section{Spinal Deformity}

In lesser resource countries the patients of TB spine generally present for the first time with spinal (kyphotic) deformity. The spinal deformity either remains static or increase while on treatment. If the kyphotic deformity heals with 60 degrees or more, the patient may present later with late onset paraplegia (paraplegia with healed disease). The surgical correction of healed kyphotic deformities is fraught with complications, hence all those patients who presented with 60 degree kyphotic deformity or likely to heal with 60 degree or more deformity should be surgically corrected in active disease. The final kyphosis can be predicted in adults before the start of treatment in active disease. Rajasekran et al proposed a formula to predict final kyphosis (11). We analysed 70 patients who were treated non-operatively $(n=40)$ and by uninstrumented surgical decompression $(n=30)$ (12). The final kyphosis recorded was correlated with initial VB height loss. The prediction of final kyphosis was better in nonoperative group. The final kyphosis of 66.5 degrees $+/-10$ degree will occur in a patient with initial VB loss (IVBL) of more than two VB height. The patient with IVBL of $1.5 \mathrm{VB}$ height or more in dorsal and dorsolumbar spine should be surgically treated by deformity correction.

\section{Surgery in Spinal TB}

The surgical decompression in patients of spinal TB with paraplegia having paretic intercostals with/without concomitant pulmonary disease is risky and requires excellent spinal surgery infrastructure. In underdeveloped countries, the infrastructure for complicated spinal surgery is sparse and disease burden is always more than available infrastructure. The Randomized controlled trial was performed where surgical decompression surgery in the dorsal spine by the transthoracic transpleural approach and extrapleural anterolateral approach were evaluated for neural 
recovery and adequacy of surgical decompression. Both the procedures showed adequate and comparable quality of surgical decompression. Besides anterior decompression concomittent posterior instrumentation can also be performed by the extrapleural anterolateral approach. "T" incision was advocated instead of semicircular incision to increase the extent of exposure for instrumented stabilization (13-15). Hence, complex transthoracic anterior decompression is now rarely advocated as surgical options in TB spine of dorsal and dorsolumbar spine.

\section{Need of Instrumented Stabilization in TB Spine}

Lots of articles were published using instrumented stabilization. All authors though have used instrumented stabilization but have not described any indication for instrumented stabilization and it was performed with the objective to prevent on treatment deterioration of the kyphotic deformity. A total of 124 published articles from 1986 to 2006 where instrumentations have been used were analysed and published (16). None of the studies have defined indications to instrumentation and the rationale for the use of type of implant. Most of the authors have performed instrumented stabilization of the spine to prevent the postsurgical deterioration of the kyphosis (16, 17). The indications of instrumented stabilizations were listed by us as: (i) grossly unstable or potentially unstable spine (panvertebral disease) to prevent pathological subluxation/dislocation and the consequent development of severe neural deficit; (ii) long segment disease- the posterior instrumentation should be performed in cases with 4 or more vertebral body affection and, junctional region TB to support anterior bone graft to bridge the gap created after decompression; and (iii) instrumentation is an essential step as a part of procedure of surgical correction of kyphotic deformity.

\section{Surgical Correction of Kyphotic Deformity}

Since the surgical correction of kyphotic deformity is a complex surgery, hence each case has to be planned for the surgical approach and steps of deformity correction. A series of patients with TB spine who presented with kyphotic deformity were treated by deformity correction by the extrapleural anterolateral approach. The issues in kyphotic deformity correction surgery of spinal TB need to be addressed are (a) retropulsed tissue in the spinal canal directly compressing the spinal cord; (b) shortened vertebral column of longstanding and spinal cord has adjusted to long standing shortened length of vertebral column. Both issues can be addressed by anterior corpectomy, and posterior column shortening. The steps for deformity correction are anterior corpectomy, posterior column shortening, instrumented posterior stabilization, and anterior and posterior bone grafting (both) performed simultaneously and sequentially. The extrapleural anterolateral approach allows all steps to be performed simultaneously with posterior Hartshill instrumentation in dorsal spine. Since the kyphotic deformity is common in dorsolumbar spine, hence, a series of patients were taken for the same procedure performed by extrapleural retroperitoneal approach for thoraco-lumbar lesions. These two approaches allow surgical correction of kyphotic deformities in less resource infrastructures $(18,19)$.

\section{Optimum Duration of Antitubercular Therapy (ATT)}

Multiple studies have provided some evidence on duration of ATT which is variable between 6/9/12/18 months. This controversy can only be resolved if the end-point of treatment is defined. Well defined end-point of treatment does not exist as most of the articles have considered clinical/clinicoradiological, and hematological criteria with no recurrence for 2 years as healed status. MRI based criteria to 
define healed status were taken by us to stop ATT in a prospective study to establish the efficacy of extended DOTS Category I of chemotherapy in spinal TB (20). Fifty two patients were evaluated by contrast MRI while on ATT; $35.2 \%$ patients attained healed status at 8 months, $60 \%$ at 12 months and $90 \%$ at 18 months. It was concluded and considered unscientific to stop ATT by fixed time schedule and we need to evaluate all spinal tubercular lesions at 8 months and subsequently to observe MRI signs of healed status before deciding stoppage of ATT. Since these patients were followed-up on contrast MRI which shows inflammation early and inflammation on MRI does not differentiate on active disease or healing disease, hence we evaluated a series of 30 patients by contrast MRI and PET scan. In an unpublished report we concluded that PET scan shows complete absence of the fluorodeoxyglucose (FDG) uptake when the TB spine lesion heals, even in the presence of contrast enhancement of the lesion on MRI. The duration of ATT to achieve healing of the lesion was found to be variable from 12 months to 24 months. This prospective study reiterated the observation of previous study that TB spine patient should be evaluated at 9,12, 18 months and lesion should not be left until it attains the healed status.

\section{Failure of Conservative Treatment}

The failure of response to ATT could be due to paradoxical reaction or drug resistance of Mycobacterium tuberculosis. We evaluated prospectively a series of therapeutically refractory cases of TB spine (21). All patients who did not show clinical improvement on ATT or the lesion or spinal deformity deteriorated while on ATT or a new lesion appeared or wound dehiscence occurred at 5 months of ATT were included. These patients were labeled as presumptive drug resistance. The lesions were debrided and tissue was submitted for BACTEC culture. Two of 14 cases only demonstrated drug resistance bacteriologically, although histological diagnosis was TB in all. The remaining patients were treated as cases of clinical drug resistance on the protocol of multidrug resistant (MDR)-TB. All patients showed healing of lesion on contrast MRI/PET scan. Since we had a poor percentage of proving drug resistance we continued to analyze another series of cases where the tissue after debridement was also submitted for Cartridgebased Nucleic Acid Amplification Technique (CB-NATT), Line Probe Assay (LPA) and Liquid Culture also. The molecular method and liquid culture improved the diagnosis of drug resistance.

\section{Atypical Presentation of Spinal TB}

Atypical spinal TB including intraspinal granuloma present with unique diagnostic challenges, hence are likely to be a missed diagnosis in initial stages of disease and later diagnosis is made when they develop complications. The intraspinal tubercular granuloma is one third of all spinal tumor syndromes in endemic region for TB. Extradural granuloma show good neural outcome after surgical decompression and peeling of granulomas, while intra-medullary granuloma respond/resolve with supervised $\operatorname{ATT}(22,23)$.

While working and analyzing TB spine during the last 28 years, we reported pseudoaneurysm of aorta (24) and written review articles highlighting the consensus achieved and listing unanswered questions in TB spine of adults and children (25-27). We received 1470 citations (source Google Scholar) on the published articles. I was also invited to be the Guest Editor for a Symposium on "Management of Spinal TB" in "Clinical Orthopedics and Related Research" which got published in July (Vol. 460), 2007 Issue (28). Later on Indian Journal of Orthopaedics also 
published a Symposium Proceedings in 2012 (29). In the recent meta-analysis performed by Wang et al (2017) on "Trends of Spinal Tuberculosis Research (1994-2015)- A Bibliometric Report", it was stated that "Dr Jain AK has published most papers in this field $(n=20)$ " (30). All the discussed research and observations lead to resolution of a number of issues by consensus on:

a) Clinicoradiological predictors to suspect and diagnose spinal TB early in the predestructive stage before a complication develops,

b) The diagnostic features of $\mathrm{TB}$ spine on MRI,

c) MRI correlation for recoverable/nonrecoverable paraplegia,

d) Define preference for surgical approaches for TB spine,

e) Indications of instrumented stabilization in TB spine,

f) Prediction of final kyphosis and correction of kyphotic deformity,

g) Treatment protocol of suspected drug resistance, and

h) Optimum duration of ATT is still under study, although 2 studies have concluded that TB spine should not be treated by fixed time frame and the lesion should be evaluated by contrast MRI and PET scan to demonstrate attainment of healed state. Currently, we are working on the role of CB-NATT and Line Probe Assay in diagnosis of spinal TB.

Working for 28 years over 3300 patients, 44 publications, and 25 research questions could contribute significantly in solving unresolved gaps in the knowledge on spinal TB. Recently, framed guidelines for treatment of bone and joint $\mathrm{TB}$ as a team lead in collaboration with the Ministry of Health and Family Welfare in association with WHO (31) and published a book on: "Tuberculosis of Bones, Joints and Spine- Evidence Based Management Guide" (32), we could give evidence on most of the issues where a very low evidence was available and highlighted the areas where research is still to be performed to meet the unmet needs of patients living with bone TB (32).

\section{Growth of Science}

To succeed as a competitive marathon runner, we have to practice over many months/years to improve cardiac fitness, musculoskel et al resilience, ability to run small distance, middle distance and long distance and then the practice to improve timing. The bigger goal is broken into small practice sessions and finally attaining fitness for competition. The run has to start with single step to complete 42 kilometers. At no point the focus of athelete should break. The same is true with growth of science. If the thought process of a researcher wavers in heterogeneous directions than effective output of the clinical solutions is very low even if a clinician is working overtime to solve multiple research questions. Although lot of research papers are published by one researcher, it still does not bring a perceptible change in clinical practice. One research question may answer a query. To change a practice one requires a series of research questions/clinical situations needing specific answers. If a clinician works slow and steady by a series of sequential research questions and by sustained and focused efforts, the research output can change the clinical practice. Mark Twain once said "The secret of getting task ahead is getting started." Break your complex overwhelming tasks into smaller manageable tasks than start working on one by one. While moving in one research direction many more new dimensions will be perceived. Success is the sum of small efforts made to answer research questions day in and day out.

\section{Acknowledgement}

The author acknowledges the contribution taken from primary publication on the same subject in Indian Journal of Orthopaedics (33). 


\section{References}

1. http://www.en:wikipedia.org/wiki/ sushruta_samhita.

2. Tuli SM (2013). Historical aspects of Pott's disease (spinal tuberculosis) management. Eur Spine J 22 (Suppl 4): 529-538.

3. Jain AK, Jena AN, Dhammi IK (1997). Syrinx formation in spinal cord in advanced tubercular quadri/paraplegia $-\mathrm{A}$ brief report. Indian JOrthop 31:136-138.

4. Jain AK, Sreenivasan R, Saini NS, Kumar S, Jain S, Dhammi IK (2012). Magnetic resonance evaluation of tubercular lesion in spine. Int Orthop 36:261-269.

5. Kamara E, Mehta S, Brust JC, Jain AK (2012). Effect of delayed diagnosis on severity of Pott's disease. Int Orthop 36:245-254.

6. Jain AK, Jena AN, Dhammi IK, Kumar S (1999). Fate of intervertebral disc space in par-adiscal tuberculous lesions. Indian $J$ Orthop 33:90-94.

7. Jain AK, Kumar S, Tuli SM (1999). Tuberculosis of spine (C1 to D4). Spinal Cord 37:362-369.

8. Jain AK, Arora A, Kumar S, Sethi A, Avtar R (1994). Measurement of prevertebral soft tissue space in cervical spine in an Indian population. Indian J Orthop 28:2731.

9. Jain AK, Jena A, Dhammi IK (2000). Correlation of clinical course with magnetic resonance imaging in tuberculous myelopathy. Neurol India 48:132-139.

10. Jain AK, Aggarwal A, Mehrotra G (1999). Correlation of canal encroachment with neurological deficit in tuberculosis of the spine. Int Orthop 23:85-86.

11. Rajasekaran S, Shanmugasundaram TK (1987). Prediction of the angle of gibbus deformity in tuberculosis of the spine. $J$ Bone Joint Surg Am 69:503-509.
12. Jain AK, Aggarwal PK, Arora A, Singh S (2004). Behaviour of the kyphotic angle in spinal tuberculosis. Int Orthop 28:110114.

13. Jain AK, Agarwal P, Singh S, Arora A (2004). Extrapleural anterolateral decompression in spinal tuberculosis. $J$ Bone Joint Surg 86-B(7):1027-1031.

14. Jain AK, Dhammi IK, Prashad B, Sinha S, Mishra P (2008). Simultaneous anterior decompression and posterior instrumentation of the tuberculous spine using an anterolateral extrapleural approach. J Bone Joint Surg (Br) 90B:1477-1481.

15. Jain AK, Dhammi IK, Jain S, Kumar J (2010). Simultaneously anterior decompression and posterior instrumentation by extrapleural retroperitoneal approach in thoracolumbar lesions. Indian J Orthop 44:409-416.

16. Jain AK, Dhammi IK (2007). Tuberculosis of the spine: A review. Clin Orthop Relat Res 460:39-49.

17. Jain AK, Jain S (2012). Instrumented stabilization in spinal tuberculosis. Int Orthop 36:285-292.

18. Jain AK, Maheshwari AV, Jena S (2007). Kyphus correction in spinal tuberculosis. Clin Orthop Relat Res 460:117-123.

19. Jain AK, Dhammi IK, Jain S, Mishra P (2010). Kyphosis in spinal tuberculosis prevention and correction. Indian $J$ Orthop 44:127-136.

20. Jain AK, Srivastava A, Saini NS, Dhammi IK, Sreenivasan R, Kumar S (2012). Efficacy of extended DOTS category I chemotherapy in spinal tuberculosis based on MRI-based healed status. Indian $J$ Orthop 46:633-639.

21. Jain AK, Dhammi IK, Modi P, Kumar J, Sreenivasan R, Saini NS (2012). Tuberculosis spine: therapeutically refractory disease. Indian J Orthop 46:171-178. 
22. Dhammi IK, Jain AK (2002). Nonoperative treatment intramedullary tuberculoma. Trop Doct 32:2-3.

23. Kumar S, Jain AK, Dhammi IK, Aggarwal AN (2007). Treatment of intraspinal tuberculoma. Clin Orthop Relat Res 460:62-66.

24. Jain AK, Chauhan RS, Dhammi IK, Maheshwari AV, Ray R (2007). Tubercular pseudoaneurysm of aorta: a rare association with vertebral tuberculosis. Spine J 7:249-253.

25. Jain AK (2010). Tuberculosis of the spine: a fresh look at an old disease. J Bone Joint Surg Br 92:905-913.

26. Jain AK, Sreenivasan R, Mukunth R, Dhammi IK (2014). Tubercular spondylitis in children. Indian J Orthop 48:136-144.

27. Jain AK, Kumar J (2013). Tuberculosis of spine: neurological deficit. Eur Spine J 22 Suppl 4:624-633.

28. Jain AK (2007). Tuberculosis of the spine. Clin Orthop Relat Res 460:2-3.
29. Jain AK, Rajasekaran S (2012). Tuberculosis of the spine. Indian J Orthop 46:127-129.

30. Wang Y, Wang Q, Zhu R, et al (2016). Trends of spinal tuberculosis research (1994-2015): a bibliometric study. Medicine (Baltimore) 95(38): e4923.

31. Anonymous (2016). Spinal TB and other forms of bone and joint TB. In: INDEXTB Guidelines: Guidelines on extrapulmonary tuberculosis for India. Initiative of Central TB Division, Ministry of Health and Family Welfare, GoI, 75-84.

32. Jain AK, Sreenivasan R, Singh N, Saha R (2017). Tuberculosis of Bones, Joints and Spine: Evidence Based Management Guide, 1st edn. New Delhi: CBS Publishers and Distributors Pvt. Ltd.

33. Jain A K (2016). Tuberculosis of spine: research evidence to treatment guidelines. Indian J Ortho 50:3-9. 